\title{
On the Solutions of a System of Third-Order Rational Difference Equations
}

\author{
A. M. Alotaibi $\left(D,{ }^{1}\right.$ M. S. M. Noorani, ${ }^{1}$ and M. A. El-Moneam ${ }^{2}$ \\ ${ }^{1}$ School of Mathematical Sciences, Universiti Kebangsaan Malaysia, Bangi, Selangor, Malaysia \\ ${ }^{2}$ Mathematics Department, Faculty of Science, Jazan University, Jazan, Saudi Arabia
}

Correspondence should be addressed to A. M. Alotaibi; ab-alo@hotmail.com

Received 21 January 2018; Accepted 20 March 2018; Published 3 May 2018

Academic Editor: Garyfalos Papashinopoulos

Copyright (C) 2018 A. M. Alotaibi et al. This is an open access article distributed under the Creative Commons Attribution License, which permits unrestricted use, distribution, and reproduction in any medium, provided the original work is properly cited.

The structure of the solutions for the system nonlinear difference equations $x_{n+1}=y_{n} y_{n-2} /\left(x_{n-1}+y_{n-2}\right), y_{n+1}=x_{n} x_{n-2} /\left( \pm y_{n-1} \pm x_{n-2}\right)$, $n=0,1, \ldots$, is clarified in which the initial conditions $x_{-2}, x_{-1}, x_{0}, y_{-2}, y_{-1}, y_{0}$ are considered as arbitrary positive real numbers. To exemplify the theoretical discussion, some numerical examples are presented.

\section{Introduction}

The prime objective of this paper is to analyze the forms of the solution of the following difference equation systems:

$$
\begin{aligned}
& x_{n+1}=\frac{y_{n} y_{n-2}}{x_{n-1}+y_{n-2}}, \\
& y_{n+1}=\frac{x_{n} x_{n-2}}{ \pm y_{n-1} \pm x_{n-2}}, \\
& \quad n=0,1, \ldots,
\end{aligned}
$$

in which the initial conditions $x_{-2}, x_{-1}, x_{0}, y_{-2}, y_{-1}, y_{0}$ are arbitrary positive real numbers.

Difference equations appear as a natural model of evolution phenomena. Furthermore, considerable findings in difference equation theory have been retrieved as discrete analogues and as numerical solutions of differential equations. This is notably true in the case of Lyapunov theory of stability. What is more, it has applications in biology, ecology, economy, physics, and so on. Due to this matter, there has been a rise in the interest in the study of qualitative analysis of scalar rational difference equations and rational system of difference equations. Although difference equations look simple in form, it is quite difficult to understand thoroughly the behaviors of their solutions because some prototypes for the development of the basic theory of the global behavior of nonlinear difference equation come from the results of rational difference equations (see [1-6]) and the references cited therein.

El-Dessoky and Elsayed [7] have analyzed the form of the solutions and the periodicity character of the following systems of rational difference equations:

$$
\begin{aligned}
& x_{n+1}=\frac{x_{n} y_{n-1}}{y_{n-1} \pm y_{n}}, \\
& y_{n+1}=\frac{y_{n} x_{n-1}}{x_{n-1} \pm x_{n}} .
\end{aligned}
$$

Elsayed and Alghamdi [8] have examined the form of the solutions of the following nonlinear difference equations systems:

$$
\begin{aligned}
& x_{n+1}=\frac{x_{n-7}}{1+x_{n-7} y_{n-3}}, \\
& y_{n+1}=\frac{y_{n-7}}{ \pm 1 \pm x_{n-3} y_{n-7}} .
\end{aligned}
$$

Elsayed et al. [9] have investigated the form of the solutions of the following difference equations systems:

$$
\begin{aligned}
& x_{n+1}=\frac{y_{n} x_{n-2}}{y_{n}+y_{n-3}}, \\
& y_{n+1}=\frac{x_{n} y_{n-2}}{ \pm x_{n} \pm x_{n-3}} .
\end{aligned}
$$


Kurbanli [10] has explored the following system of difference equations:

$$
\begin{aligned}
& x_{n+1}=\frac{x_{n-1}}{y_{n} x_{n-1}+1}, \\
& y_{n+1}=\frac{y_{n-1}}{x_{n} y_{n-1}+1} .
\end{aligned}
$$

Mansour et al. [11] have got the form of the solutions of some systems of the following rational difference equations:

$$
\begin{aligned}
& x_{n+1}=\frac{x_{n-1}}{\alpha-x_{n-1} y_{n}}, \\
& y_{n+1}=\frac{y_{n-1}}{\beta+\gamma y_{n-1} x_{n}} .
\end{aligned}
$$

Touafek and Elsayed [12] have studied the periodicity and gave the form of the solutions of the following systems of difference equations of order two:

$$
\begin{aligned}
& x_{n+1}=\frac{y_{n} x_{n-1}}{ \pm x_{n-1} \pm y_{n}}, \\
& y_{n+1}=\frac{x_{n} y_{n-1}}{ \pm x_{n} \pm x_{n-1}} .
\end{aligned}
$$

Equations similar to difference equations and nonlinear systems of rational difference equations were investigated; see [13-20].

\section{Main Results}

2.1. The First System: $x_{n+1}=y_{n} y_{n-2} /\left(x_{n-1}+y_{n-2}\right), y_{n+1}=$ $x_{n} x_{n-2} /\left(y_{n-1}+x_{n-2}\right)$. The solutions of the system of two difference equations are analyzed in this subsection.

$$
\begin{aligned}
& x_{n+1}=\frac{y_{n} y_{n-2}}{x_{n-1}+y_{n-2}}, \\
& y_{n+1}=\frac{x_{n} x_{n-2}}{y_{n-1}+x_{n-2}}, \\
& \quad n=0,1, \ldots,
\end{aligned}
$$

where the initial conditions $x_{-2}, x_{-1}, x_{0}, y_{-2}, y_{-1}, y_{0}$ are arbitrary positive real numbers.

Theorem 1. Suppose that $\left\{x_{n}, y_{n}\right\}$ are solutions of system (8). Then for $n=0,1,2, \ldots$, one has

$$
\begin{aligned}
x_{2 n-1} & =\frac{b d f}{\left(f_{n-2} d+f_{n-1} b\right)\left(f_{n-1} b+f_{n} f\right)}, \\
x_{2 n} & =\frac{a c e}{\left(f_{n-1} a+f_{n} e\right)\left(f_{n-1} e+f_{n} c\right)}, \\
y_{2 n-1} & =\frac{a c e}{\left(f_{n-2} a+f_{n-1} e\right)\left(f_{n-1} e+f_{n} c\right)}, \\
y_{2 n} & =\frac{b d f}{\left(f_{n-1} b+f_{n} f\right)\left(f_{n-1} d+f_{n} b\right)},
\end{aligned}
$$

where $x_{0}=a, x_{-1}=b, x_{-2}=c, y_{0}=d, y_{-1}=e, y_{-2}=f$ and where $f_{n}$ is the Fibonacci Sequence with $f_{-2}=1, f_{-1}=0$.

Proof. For $n=0$, the result holds. By using mathematical induction now suppose that $n>0$ and that our assumption holds for $-1, n-2$. That is,

$$
\begin{aligned}
& x_{2 n-4}=\frac{a c e}{\left(f_{n-3} a+f_{n-2} e\right)\left(f_{n-3} e+f_{n-2} c\right)}, \\
& x_{2 n-3}=\frac{b d f}{\left(f_{n-3} d+f_{n-2} b\right)\left(f_{n-2} b+f_{n-1} f\right)}, \\
& x_{2 n-2}=\frac{a c e}{\left(f_{n-2} a+f_{n-1} e\right)\left(f_{n-2} e+f_{n-1} c\right)}, \\
& y_{2 n-4}=\frac{b d f}{\left(f_{n-3} b+f_{n-2} f\right)\left(f_{n-3} d+f_{n-2} b\right)}, \\
& y_{2 n-3}=\frac{a c e}{\left(f_{n-3} a+f_{n-2} e\right)\left(f_{n-2} e+f_{n-1} c\right)}, \\
& y_{2 n-2}=\frac{b d f}{\left(f_{n-2} b+f_{n-1} f\right)\left(f_{n-2} d+f_{n-1} b\right)} .
\end{aligned}
$$

Now, it follows from system (8) substitution of the above equations that

$$
\begin{aligned}
x_{2 n-1} & =\frac{y_{2 n-2} y_{2 n-4}}{x_{2 n-3}+y_{2 n-4}}=\frac{\left(b d f /\left(f_{n-2} b+f_{n-1} f\right)\left(f_{n-2} d+f_{n-1} b\right)\right)\left(b d f /\left(f_{n-3} b+f_{n-2} f\right)\left(f_{n-3} d+f_{n-2} b\right)\right)}{b d f /\left(f_{n-3} d+f_{n-2} b\right)\left(f_{n-2} b+f_{n-1} f\right)+b d f /\left(f_{n-3} b+f_{n-2} f\right)\left(f_{n-3} d+f_{n-2} b\right)} \\
& =\frac{\left(b d f /\left(f_{n-2} b+f_{n-1} f\right)\left(f_{n-2} d+f_{n-1} b\right)\right)\left(1 /\left(f_{n-3} b+f_{n-2} f\right)\right)}{1 /\left(f_{n-2} b+f_{n-1} f\right)+1 /\left(f_{n-3} b+f_{n-2} f\right)} \\
& =\frac{b d f /\left(f_{n-2} d+f_{n-1} b\right)}{\left(f_{n-2} b+f_{n-1} f\right)\left(f_{n-3} b+f_{n-2} f\right)\left[1 /\left(f_{n-2} b+f_{n-1} f\right)+1 /\left(f_{n-3} b+f_{n-2} f\right)\right]}=\frac{b d f /\left(f_{n-2} d+f_{n-1} b\right)}{\left[f_{n-3} b+f_{n-2} f+f_{n-2} b+f_{n-1} f\right]} \\
& =\frac{b d f /\left(f_{n-2} d+f_{n-1} b\right)}{\left[f_{n-1} b+f_{n} f\right]}=\frac{b d f}{\left(f_{n-2} d+f_{n-1} b\right)\left(f_{n-1} b+f_{n} f\right)}, \\
y_{2 n-1} & =\frac{x_{2 n-2} x_{2 n-4}}{y_{2 n-3}+x_{2 n-4}}=\frac{\left(a c e /\left(f_{n-2} a+f_{n-1} e\right)\left(f_{n-2} e+f_{n-1} c\right)\right)\left(\operatorname{ace} /\left(f_{n-3} a+f_{n-2} e\right)\left(f_{n-3} e+f_{n-2} c\right)\right)}{a c e /\left(f_{n-3} a+f_{n-2} e\right)\left(f_{n-2} e+f_{n-1} c\right)+\operatorname{ace} /\left(f_{n-3} a+f_{n-2} e\right)\left(f_{n-3} e+f_{n-2} c\right)}
\end{aligned}
$$




$$
\begin{aligned}
& =\frac{\left(a c e /\left(f_{n-2} a+f_{n-1} e\right)\left(f_{n-2} e+f_{n-1} c\right)\right)\left(1 /\left(f_{n-3} e+f_{n-2} c\right)\right)}{1 /\left(f_{n-2} e+f_{n-1} c\right)+1 /\left(f_{n-3} e+f_{n-2} c\right)} \\
& =\frac{a c e /\left(f_{n-2} a+f_{n-1} e\right)}{\left(f_{n-2} e+f_{n-1} c\right)\left(f_{n-3} e+f_{n-2} c\right)\left[1 /\left(f_{n-2} e+f_{n-1} c\right)+1 /\left(f_{n-3} e+f_{n-2} c\right)\right]}=\frac{a c e /\left(f_{n-2} a+f_{n-1} e\right)}{\left[f_{n-3} e+f_{n-2} c+f_{n-2} e+f_{n-1} c\right]} \\
& =\frac{a c e /\left(f_{n-2} a+f_{n-1} e\right)}{\left[f_{n-1} e+f_{n} c\right]}=\frac{a c e}{\left(f_{n-2} a+f_{n-1} e\right)\left(f_{n-1} e+f_{n} c\right)} .
\end{aligned}
$$

Also, we obtain

$$
\begin{aligned}
x_{2 n} & =\frac{y_{2 n-1} y_{2 n-3}}{x_{2 n-2}+y_{2 n-3}}=\frac{\left(a c e /\left(f_{n-2} a+f_{n-1} e\right)\left(f_{n-1} e+f_{n} c\right)\right)\left(a c e /\left(f_{n-3} a+f_{n-2} e\right)\left(f_{n-2} e+f_{n-1} c\right)\right)}{a c e /\left(f_{n-2} a+f_{n-1} e\right)\left(f_{n-2} e+f_{n-1} c\right)+a c e /\left(f_{n-3} a+f_{n-2} e\right)\left(f_{n-2} e+f_{n-1} c\right)} \\
& =\frac{\left(a c e /\left(f_{n-2} a+f_{n-1} e\right)\left(f_{n-1} e+f_{n} c\right)\right)\left(1 /\left(f_{n-3} a+f_{n-2} e\right)\right)}{1 /\left(f_{n-2} a+f_{n-1} e\right)+1 /\left(f_{n-3} a+f_{n-2} e\right)} \\
& =\frac{a c e /\left(f_{n-1} e+f_{n} c\right)}{\left(f_{n-2} a+f_{n-1} e\right)\left(f_{n-3} a+f_{n-2} e\right)\left[1 /\left(f_{n-2} a+f_{n-1} e\right)+1 /\left(f_{n-3} a+f_{n-2} e\right)\right]}=\frac{a c e /\left(f_{n-1} e+f_{n} c\right)}{\left[f_{n-3} a+f_{n-2} e+f_{n-2} a+f_{n-1} e\right]} \\
& =\frac{a c e /\left(f_{n-1} e+f_{n} c\right)}{\left[f_{n-1} a+f_{n} e\right]}=\frac{a c e}{\left(f_{n-1} a+f_{n} e\right)\left(f_{n-1} e+f_{n} c\right)}, \\
y_{2 n} & =\frac{x_{2 n-1} x_{2 n-3}}{y_{2 n-2}+x_{2 n-3}}=\frac{\left(b d f /\left(f_{n-2} d+f_{n-1} b\right)\left(f_{n-1} b+f_{n} f\right)\right)\left(b d f /\left(f_{n-3} d+f_{n-2} b\right)\left(f_{n-2} b+f_{n-1} f\right)\right)}{b d f /\left(f_{n-2} b+f_{n-1} f\right)\left(f_{n-2} d+f_{n-1} b\right)+b d f /\left(f_{n-3} d+f_{n-2} b\right)\left(f_{n-2} b+f_{n-1} f\right)} \\
& =\frac{\left(b d f /\left(f_{n-2} d+f_{n-1} b\right)\left(f_{n-1} b+f_{n} f\right)\right)\left(1 /\left(f_{n-3} d+f_{n-2} b\right)\right)}{1 /\left(f_{n-2} d+f_{n-1} b\right)+1 /\left(f_{n-3} d+f_{n-2} b\right)} \\
& =\frac{\left(f_{n-2} d+f_{n-1} b\right)\left(f_{n-3} d+f_{n-2} b\right)\left[1 /\left(f_{n-2} d+f_{n-1} b\right)+1 /\left(f_{n-3} d+f_{n-2} b\right)\right]}{\left(f_{n-3} d+f_{n-2} b+f_{n-2} d+f_{n-1} b\right]} \\
& =\frac{b d f /\left(f_{n-1} b+f_{n} f\right)}{\left[f_{n-1} d+f_{n} b\right]}=\frac{b d f}{\left(f_{n-1} b+f_{n} f\right)\left(f_{n-1} d+f_{n} b\right)} .
\end{aligned}
$$

Hence the proof is complete.

Lemma 2. Let $\left\{x_{n}, y_{n}\right\}$ be a positive solution of system (8); then every solution of system (8) is bounded and converges to zero.

Proof. It follows from system (8) that

$$
\begin{aligned}
& x_{n+1}=\frac{y_{n} y_{n-2}}{x_{n-1}+y_{n-2}} \leq \frac{y_{n} y_{n-2}}{y_{n-2}} \leq y_{n}, \\
& y_{n+1}=\frac{x_{n} x_{n-2}}{y_{n-1}+x_{n-2}} \leq \frac{x_{n} x_{n-2}}{x_{n-2}} \leq x_{n} ;
\end{aligned}
$$

thus

$$
\begin{aligned}
& x_{n+1} \leq y_{n} \leq x_{n-1}, \\
& y_{n+1} \leq x_{n} \leq y_{n-1} .
\end{aligned}
$$

Then the subsequences $\left\{x_{2 n-1}\right\}_{n=0}^{\infty},\left\{x_{2 n}\right\}_{n=0}^{\infty}$ are decreasing and so are bounded from above by

$$
M=\max \left\{x_{-2}, x_{-1}, x_{0}\right\} .
$$

Similarly the subsequences $\left\{y_{2 n-1}\right\}_{n=0}^{\infty},\left\{y_{2 n}\right\}_{n=0}^{\infty}$ are decreasing and so are bounded from above by

$$
N=\max \left\{y_{-2}, y_{-1}, y_{0}\right\} \text {. }
$$

2.2. The Second System: $x_{n+1}=y_{n} y_{n-2} /\left(x_{n-1}+y_{n-2}\right), y_{n+1}=$ $x_{n} x_{n-2} /\left(y_{n-1}-x_{n-2}\right)$. The structure of the solutions of the following system of the difference equations is examined in this subsection.

$$
\begin{aligned}
& x_{n+1}=\frac{y_{n} y_{n-2}}{x_{n-1}+y_{n-2}}, \\
& y_{n+1}=\frac{x_{n} x_{n-2}}{y_{n-1}-x_{n-2}},
\end{aligned}
$$

$$
n=0,1, \ldots,
$$

with nonzero positive real numbers of initial conditions $x_{-2}$, $x_{-1}, x_{0}$ and $y_{-2}, y_{-1}, y_{0}$ with $x_{-2} \neq y_{-1}$. 
Theorem 3. Suppose that $\left\{x_{n}, y_{n}\right\}$ are solutions of the system

$$
\begin{aligned}
& x_{n+1}=\frac{y_{n} y_{n-2}}{x_{n-1}+y_{n-2}}, \\
& y_{n+1}=\frac{x_{n} x_{n-2}}{y_{n-1}-x_{n-2}} .
\end{aligned}
$$

Then for $n=0,1,2, \ldots$, one has

$$
\begin{aligned}
x_{2 n-1} & =\frac{(-1)^{n} b d f}{\left(f_{n-2} d-f_{n-1} b\right)\left(f_{n-1} b+f_{n} f\right)}, \\
x_{2 n} & =\frac{(-1)^{n+1} a c e}{\left(f_{n-1} a+f_{n} e\right)\left(f_{n-1} e-f_{n} c\right)}, \\
y_{2 n-1} & =\frac{(-1)^{n+1} a c e}{\left(f_{n-2} a+f_{n-1} e\right)\left(f_{n-1} e-f_{n} c\right)}, \\
y_{2 n} & =\frac{(-1)^{n+1} b d f}{\left(f_{n-1} b+f_{n} f\right)\left(f_{n-1} d-f_{n} b\right)},
\end{aligned}
$$

where $x_{0}=a, x_{-1}=b, x_{-2}=c, y_{0}=d, y_{-1}=e, y_{-2}=f$ and where $f_{n}$ is the Fibonacci Sequence with $f_{-2}=1, f_{-1}=0$.
Proof. For $n=0$ the result holds. By using mathematical induction now suppose that $n>0$ and that our assumption holds for $n-1, n-2$. That is,

$$
\begin{aligned}
& x_{2 n-4}=\frac{(-1)^{n-1} a c e}{\left(f_{n-3} a+f_{n-2} e\right)\left(f_{n-3} e-f_{n-2} c\right)}, \\
& x_{2 n-3}=\frac{(-1)^{n-1} b d f}{\left(f_{n-3} d-f_{n-2} b\right)\left(f_{n-2} b+f_{n-1} f\right)}, \\
& x_{2 n-2}=\frac{(-1)^{n} a c e}{\left(f_{n-2} a+f_{n-1} e\right)\left(f_{n-2} e-f_{n-1} c\right)}, \\
& y_{2 n-4}=\frac{(-1)^{n-1} b d f}{\left(f_{n-3} b+f_{n-2} f\right)\left(f_{n-3} d-f_{n-2} b\right)}, \\
& y_{2 n-3}=\frac{(-1)^{n} a c e}{\left(f_{n-3} a+f_{n-2} e\right)\left(f_{n-2} e-f_{n-1} c\right)}, \\
& y_{2 n-2}=\frac{(-1)^{n} b d f}{\left(f_{n-2} b+f_{n-1} f\right)\left(f_{n-2} d-f_{n-1} b\right)} .
\end{aligned}
$$

Now, it follows from system (17) substitution of the above equations that

$$
\begin{aligned}
x_{2 n-1} & =\frac{y_{2 n-2} y_{2 n-4}}{x_{2 n-3}+y_{2 n-4}}=\frac{\left((-1)^{n} b d f /\left(f_{n-2} b+f_{n-1} f\right)\left(f_{n-2} d-f_{n-1} b\right)\right)\left((-1)^{n-1} b d f /\left(f_{n-3} b+f_{n-2} f\right)\left(f_{n-3} d-f_{n-2} b\right)\right)}{(-1)^{n-1} b d f /\left(f_{n-3} d-f_{n-2} b\right)\left(f_{n-2} b+f_{n-1} f\right)+(-1)^{n-1} b d f /\left(f_{n-3} b+f_{n-2} f\right)\left(f_{n-3} d-f_{n-2} b\right)} \\
& =\frac{\left((-1)^{n} b d f /\left(f_{n-2} b+f_{n-1} f\right)\left(f_{n-2} d-f_{n-1} b\right)\right)\left(1 /\left(f_{n-3} b+f_{n-2} f\right)\right)}{1 /\left(f_{n-2} b+f_{n-1} f\right)+1 /\left(f_{n-3} b+f_{n-2} f\right)}=\frac{(-1)^{n} b d f /\left(f_{n-2} d-f_{n-1} b\right)}{\left(f_{n-3} b+f_{n-2} f\right)+\left(f_{n-2} b+f_{n-1} f\right)} \\
& =\frac{(-1)^{n} b d f}{\left(f_{n-2} d-f_{n-1} b\right)\left(f_{n-1} b+f_{n} f\right)}, \\
y_{2 n-1} & =\frac{x_{2 n-2} x_{2 n-4}}{y_{2 n-3}-x_{2 n-4}}=\frac{\left((-1)^{n} a c e /\left(f_{n-2} a+f_{n-1} e\right)\left(f_{n-2} e-f_{n-1} c\right)\right)\left((-1)^{n-1} a c e /\left(f_{n-3} a+f_{n-2} e\right)\left(f_{n-3} e-f_{n-2} c\right)\right)}{(-1)^{n} a c e /\left(f_{n-3} a+f_{n-2} e\right)\left(f_{n-2} e-f_{n-1} c\right)-(-1)^{n-1} a c e /\left(f_{n-3} a+f_{n-2} e\right)\left(f_{n-3} e-f_{n-2} c\right)} \\
& =\frac{\left((-1)^{n+1} a c e /\left(f_{n-2} a+f_{n-1} e\right)\left(f_{n-2} e-f_{n-1} c\right)\right)\left(1 /\left(f_{n-3} e-f_{n-2} c\right)\right)}{1 /\left(f_{n-2} e-f_{n-1} c\right)+1 /\left(f_{n-3} e-f_{n-2} c\right)} \\
& =\frac{(-1)^{n+1} a c e /\left(f_{n-2} a+f_{n-1} e\right)}{\left(f_{n-2} e-f_{n-1} c\right)\left(f_{n-3} e-f_{n-2} c\right)\left[1 /\left(f_{n-2} e-f_{n-1} c\right)+1 /\left(f_{n-3} e-f_{n-2} c\right)\right]}=\frac{(-1)^{n+1} a c e /\left(f_{n-2} a+f_{n-1} e\right)}{\left[f_{n-3} e-f_{n-2} c+f_{n-2} e-f_{n-1} c\right]} \\
& =\frac{(-1)^{n+1} a c e /\left(f_{n-2} a+f_{n-1} e\right)}{\left[f_{n-1} e-f_{n} c\right]}=\frac{(-1)^{n+1} a c e}{\left(f_{n-2} a+f_{n-1} e\right)\left(f_{n-1} e-f_{n} c\right)} .
\end{aligned}
$$

Also, we obtain

$$
\begin{aligned}
x_{2 n} & =\frac{y_{2 n-1} y_{2 n-3}}{x_{2 n-2}+y_{2 n-3}}=\frac{\left((-1)^{n+1} \text { ace } /\left(f_{n-2} a+f_{n-1} e\right)\left(f_{n-1} e-f_{n} c\right)\right)\left((-1)^{n} \text { ace } /\left(f_{n-3} a+f_{n-2} e\right)\left(f_{n-2} e-f_{n-1} c\right)\right)}{(-1)^{n} \text { ace } /\left(f_{n-2} a+f_{n-1} e\right)\left(f_{n-2} e-f_{n-1} c\right)+(-1)^{n} \text { ace } /\left(f_{n-3} a+f_{n-2} e\right)\left(f_{n-2} e-f_{n-1} c\right)} \\
& =\frac{\left((-1)^{n+1} \text { ace } /\left(f_{n-2} a+f_{n-1} e\right)\left(f_{n-1} e-f_{n} c\right)\right)\left(1 /\left(f_{n-3} a+f_{n-2} e\right)\right)}{1 /\left(f_{n-2} a+f_{n-1} e\right)+1 /\left(f_{n-3} a+f_{n-2} e\right)}
\end{aligned}
$$




$$
\begin{aligned}
& =\frac{(-1)^{n+1} a c e /\left(f_{n-1} e-f_{n} c\right)}{\left(f_{n-2} a+f_{n-1} e\right)\left(f_{n-3} a+f_{n-2} e\right)\left[1 /\left(f_{n-2} a+f_{n-1} e\right)+1 /\left(f_{n-3} a+f_{n-2} e\right)\right]}=\frac{(-1)^{n+1} \text { ace } /\left(f_{n-1} e-f_{n} c\right)}{\left[f_{n-3} a+f_{n-2} e+f_{n-2} a+f_{n-1} e\right]} \\
& =\frac{(-1)^{n+1} a c e /\left(f_{n-1} e-f_{n} c\right)}{\left[f_{n-1} a+f_{n} e\right]}=\frac{(-1)^{n+1} a c e}{\left(f_{n-1} a+f_{n} e\right)\left(f_{n-1} e-f_{n} c\right)}, \\
y_{2 n} & =\frac{x_{2 n-1} x_{2 n-3}}{y_{2 n-2}-x_{2 n-3}}=\frac{\left((-1)^{n} b d f /\left(f_{n-2} d-f_{n-1} b\right)\left(f_{n-1} b+f_{n} f\right)\right)\left((-1)^{n-1} b d f /\left(f_{n-3} d-f_{n-2} b\right)\left(f_{n-2} b+f_{n-1} f\right)\right)}{(-1)^{n} b d f /\left(f_{n-2} b+f_{n-1} f\right)\left(f_{n-2} d-f_{n-1} b\right)-(-1)^{n-1} b d f /\left(f_{n-3} d-f_{n-2} b\right)\left(f_{n-2} b+f_{n-1} f\right)} \\
& =\frac{\left((-1)^{n+1} b d f /\left(f_{n-2} d-f_{n-1} b\right)\left(f_{n-1} b+f_{n} f\right)\right)\left(1 /\left(f_{n-3} d-f_{n-2} b\right)\right)}{1 /\left(f_{n-2} d-f_{n-1} b\right)+1 /\left(f_{n-3} d-f_{n-2} b\right)} \\
& =\frac{(-1)^{n+1} b d f /\left(f_{n-1} b+f_{n} f\right)}{\left(f_{n-2} d-f_{n-1} b\right)\left(f_{n-3} d-f_{n-2} b\right)\left[1 /\left(f_{n-2} d-f_{n-1} b\right)+1 /\left(f_{n-3} d-f_{n-2} b\right)\right]}=\frac{(-1)^{n+1} b d f /\left(f_{n-1} b+f_{n} f\right)}{\left[f_{n-3} d-f_{n-2} b+f_{n-2} d-f_{n-1} b\right]} \\
& =\frac{(-1)^{n+1} b d f /\left(f_{n-1} b+f_{n} f\right)}{\left[f_{n-1} d-f_{n} b\right]}=\frac{(-1)^{n+1} b d f}{\left(f_{n-1} b+f_{n} f\right)\left(f_{n-1} d-f_{n} b\right)} .
\end{aligned}
$$

Hence the proof is complete.

2.3. The Third System: $x_{n+1}=y_{n} y_{n-2} /\left(x_{n-1}+y_{n-2}\right), y_{n+1}=$ $x_{n} x_{n-2} /\left(-y_{n-1}+x_{n-2}\right)$. In this subsection, the framework of the solution of the following system of difference equations is acquired.

$$
\begin{aligned}
& x_{n+1}=\frac{y_{n} y_{n-2}}{x_{n-1}+y_{n-2}}, \\
& y_{n+1}=\frac{x_{n} x_{n-2}}{-y_{n-1}+x_{n-2}}, \\
& n=0,1, \ldots,
\end{aligned}
$$

where the initial conditions $x_{-2}, x_{-1}, x_{0}, y_{-2}, y_{-1}, y_{0}$ are arbitrary positive real numbers with $x_{-2} \neq y_{-1}$.

Theorem 4. Suppose that $\left\{x_{n}, y_{n}\right\}$ are solutions of system (23). Then for $n=0,1,2, \ldots$, one sees that all solutions of system (23) are given by the following formulas:

$$
\begin{aligned}
x_{6 n-2} & =\frac{(-1)^{n} a c}{\left(f_{3 n-2} a+f_{3 n-1} e\right)}, \\
x_{6 n-1} & =\frac{(-1)^{n} b f}{\left(f_{3 n-1} b+f_{3 n} f\right)}, \\
x_{6 n} & =\frac{(-1)^{n} a e}{\left(f_{3 n-1} a+f_{3 n} e\right)}, \\
x_{6 n+1} & =\frac{(-1)^{n} d f}{\left(f_{3 n} b+f_{3 n+1} f\right)}, \\
x_{6 n+2} & =\frac{(-1)^{n} a c e}{(c-e)\left(f_{3 n} a+f_{3 n+1} e\right)},
\end{aligned}
$$

$$
\begin{aligned}
x_{6 n+3} & =\frac{(-1)^{n} b d f}{(b-d)\left(f_{3 n+1} b+f_{3 n+2} f\right)}, \\
y_{6 n-2} & =\frac{(-1)^{n} b f}{\left(f_{3 n-2} b+f_{3 n-1} f\right)}, \\
y_{6 n-1} & =\frac{(-1)^{n} a e}{\left(f_{3 n-2} a+f_{3 n-1} e\right)}, \\
y_{6 n} & =\frac{(-1)^{n} d f}{\left(f_{3 n-1} b+f_{3 n} f\right)}, \\
y_{6 n+1} & =\frac{(-1)^{n} a c e}{(c-e)\left(f_{3 n-1} a+f_{3 n} e\right)}, \\
y_{6 n+2} & =\frac{(-1)^{n} b d f}{(b-d)\left(f_{3 n} b+f_{3 n+1} f\right)}, \\
y_{6 n+3} & =\frac{(-1)^{n+1} a c}{\left(f_{3 n} a+f_{3 n+1} e\right)},
\end{aligned}
$$

where $x_{0}=a, x_{-1}=b, x_{-2}=c, y_{0}=d, y_{-1}=e, y_{-2}=f$ and where $f_{n}$ is the Fibonacci Sequence with $f_{-2}=1, f_{-1}=0$.

Proof. For $n=0$ the result holds. By using mathematical induction now suppose that $n>0$ and that our assumption holds for $n-1$. That is,

$$
\begin{aligned}
& x_{6 n-8}=\frac{(-1)^{n-1} a c}{\left(f_{3 n-5} a+f_{3 n-4} e\right)}, \\
& x_{6 n-7}=\frac{(-1)^{n-1} b f}{\left(f_{3 n-4} b+f_{3 n-3} f\right)}, \\
& x_{6 n-6}=\frac{(-1)^{n-1} a e}{\left(f_{3 n-4} a+f_{3 n-3} e\right)},
\end{aligned}
$$




$$
\begin{aligned}
& x_{6 n-5}=\frac{(-1)^{n-1} d f}{\left(f_{3 n-3} b+f_{3 n-2} f\right)}, \\
& x_{6 n-4}=\frac{(-1)^{n-1} a c e}{(c-e)\left(f_{3 n-3} a+f_{3 n-2} e\right)}, \\
& x_{6 n-3}=\frac{(-1)^{n-1} b d f}{(b-d)\left(f_{3 n-2} b+f_{3 n-1} f\right)}, \\
& y_{6 n-8}=\frac{(-1)^{n-1} b f}{\left(f_{3 n-5} b+f_{3 n-4} f\right)}, \\
& y_{6 n-7}=\frac{(-1)^{n-1} a e}{\left(f_{3 n-5} a+f_{3 n-4} e\right)},
\end{aligned}
$$$$
y_{6 n-6}=\frac{(-1)^{n-1} d f}{\left(f_{3 n-4} b+f_{3 n-3} f\right)} \text {, }
$$

Now, it follows from system (23) substitution of the above equations that

$$
\begin{aligned}
& x_{6 n-2}=\frac{y_{6 n-3} y_{6 n-5}}{x_{6 n-4}+y_{6 n-5}}=\frac{\left((-1)^{n} a c /\left(f_{3 n-3} a+f_{3 n-2} e\right)\right)\left((-1)^{n-1} a c e /(c-e)\left(f_{3 n-4} a+f_{3 n-3} e\right)\right)}{(-1)^{n-1} a c e /(c-e)\left(f_{3 n-3} a+f_{3 n-2} e\right)+(-1)^{n-1} a c e /(c-e)\left(f_{3 n-4} a+f_{3 n-3} e\right)} \\
& =\frac{\left((-1)^{n} a c /\left(f_{3 n-3} a+f_{3 n-2} e\right)\right)\left(1 /\left(f_{3 n-4} a+f_{3 n-3} e\right)\right)}{1 /\left(f_{3 n-3} a+f_{3 n-2} e\right)+1 /\left(f_{3 n-4} a+f_{3 n-3} e\right)}=\frac{(-1)^{n} a c}{\left(f_{3 n-3} a+f_{3 n-2} e\right)\left(f_{3 n-4} a+f_{3 n-3} e\right)\left[1 /\left(f_{3 n-3} a+f_{3 n-2} e\right)+1 /\left(f_{3 n-4} a+f_{3 n-3} e\right)\right]} \\
& =\frac{(-1)^{n} a c}{\left[\left(f_{3 n-4} a+f_{3 n-3} e\right)+\left(f_{3 n-3} a+f_{3 n-2} e\right)\right]}=\frac{(-1)^{n} a c}{\left(f_{3 n-2} a+f_{3 n-1} e\right)} \text {, } \\
& y_{6 n-2}=\frac{x_{6 n-3} x_{6 n-5}}{-y_{6 n-4}+x_{6 n-5}}=\frac{\left((-1)^{n-1} b d f /(b-d)\left(f_{3 n-2} b+f_{3 n-1} f\right)\right)\left((-1)^{n-1} d f /\left(f_{3 n-3} b+f_{3 n-2} f\right)\right)}{-(-1)^{n-1} b d f /(b-d)\left(f_{3 n-3} b+f_{3 n-2} f\right)+(-1)^{n-1} d f /\left(f_{3 n-3} b+f_{3 n-2} f\right)} \\
& =\frac{\left((-1)^{n-1} b d f /(b-d)\left(f_{3 n-2} b+f_{3 n-1} f\right)\right)\left(1 /\left(f_{3 n-3} b+f_{3 n-2} f\right)\right)}{-b /(b-d)\left(f_{3 n-3} b+f_{3 n-2} f\right)+1 /\left(f_{3 n-3} b+f_{3 n-2} f\right)}=\frac{(-1)^{n-1} b d f /(b-d)\left(f_{3 n-2} b+f_{3 n-1} f\right)}{-b /(b-d)+1} \\
& =\frac{(-1)^{n-1} b d f}{(b-d)\left(f_{3 n-2} b+f_{3 n-1} f\right)[-b /(b-d)+1]}=\frac{(-1)^{n-1} b d f}{\left(f_{3 n-2} b+f_{3 n-1} f\right)[-b+b-d]}=\frac{(-1)^{n-1} b d f}{\left(f_{3 n-2} b+f_{3 n-1} f\right)[-b+b-d]}=\frac{(-1)^{n} b f}{\left(f_{3 n-2} b+f_{3 n-1} f\right)}, \\
& x_{6 n}=\frac{y_{6 n-1} y_{6 n-3}}{x_{6 n-2}+y_{6 n-3}}=\frac{\left((-1)^{n} a e /\left(f_{3 n-2} a+f_{3 n-1} e\right)\right)\left((-1)^{n} a c /\left(f_{3 n-3} a+f_{3 n-2} e\right)\right)}{(-1)^{n} a c /\left(f_{3 n-2} a+f_{3 n-1} e\right)+(-1)^{n} a c /\left(f_{3 n-3} a+f_{3 n-2} e\right)}=\frac{\left((-1)^{n} a e /\left(f_{3 n-2} a+f_{3 n-1} e\right)\right)\left(1 /\left(f_{3 n-3} a+f_{3 n-2} e\right)\right)}{1 /\left(f_{3 n-2} a+f_{3 n-1} e\right)+1 /\left(f_{3 n-3} a+f_{3 n-2} e\right)} \\
& =\frac{(-1)^{n} a e}{\left(f_{3 n-2} a+f_{3 n-1} e\right)\left(f_{3 n-3} a+f_{3 n-2} e\right)\left[1 /\left(f_{3 n-2} a+f_{3 n-1} e\right)+1 /\left(f_{3 n-3} a+f_{3 n-2} e\right)\right]}=\frac{(-1)^{n} a e}{\left[f_{3 n-3} a+f_{3 n-2} e+f_{3 n-2} a+f_{3 n-1} e\right]} \\
& =\frac{(-1)^{n} a e}{\left(f_{3 n-1} a+f_{3 n} e\right)}, \\
& y_{6 n}=\frac{x_{6 n-1} x_{6 n-3}}{-y_{6 n-2}+x_{6 n-3}}=\frac{\left((-1)^{n} b f /\left(f_{3 n-1} b+f_{3 n} f\right)\right)\left((-1)^{n-1} b d f /(b-d)\left(f_{3 n-2} b+f_{3 n-1} f\right)\right)}{-(-1)^{n} b f /\left(f_{3 n-2} b+f_{3 n-1} f\right)+(-1)^{n-1} b d f /(b-d)\left(f_{3 n-2} b+f_{3 n-1} f\right)} \\
& =\frac{\left((-1)^{n} b f /\left(f_{3 n-1} b+f_{3 n} f\right)\right)\left(d /(b-d)\left(f_{3 n-2} b+f_{3 n-1} f\right)\right)}{1 /\left(f_{3 n-2} b+f_{3 n-1} f\right)+d /(b-d)\left(f_{3 n-2} b+f_{3 n-1} f\right)} \\
& =\frac{(-1)^{n} b d f}{(b-d)\left(f_{3 n-2} b+f_{3 n-1} f\right)\left(f_{3 n-1} b+f_{3 n} f\right)\left[1 /\left(f_{3 n-2} b+f_{3 n-1} f\right)+d /(b-d)\left(f_{3 n-2} b+f_{3 n-1} f\right)\right]} \\
& =\frac{(-1)^{n} b d f}{\left[(b-d)\left(f_{3 n-2} b+f_{3 n-1} f\right)\left(f_{3 n-1} b+f_{3 n} f\right) /\left(f_{3 n-2} b+f_{3 n-1} f\right)+d(b-d)\left(f_{3 n-2} b+f_{3 n-1} f\right)\left(f_{3 n-1} b+f_{3 n} f\right) /(b-d)\left(f_{3 n-2} b+f_{3 n-1} f\right)\right]} \\
& =\frac{(-1)^{n} b d f}{\left[(b-d)\left(f_{3 n-1} b+f_{3 n} f\right)+d\left(f_{3 n-1} b+f_{3 n} f\right)\right]}=\frac{(-1)^{n} b d f}{\left[b\left(f_{3 n-1} b+f_{3 n} f\right)-d\left(f_{3 n-1} b+f_{3 n} f\right)+d\left(f_{3 n-1} b+f_{3 n} f\right)\right]}=\frac{(-1)^{n} b d f}{b\left(f_{3 n-1} b+f_{3 n} f\right)} \\
& =\frac{(-1)^{n} d f}{\left(f_{3 n-1} b+f_{3 n} f\right)} \text {. }
\end{aligned}
$$


Also, we obtain

$$
\begin{aligned}
x_{6 n+2} & =\frac{y_{6 n+1} y_{6 n-1}}{x_{6 n}+y_{6 n-1}}=\frac{\left((-1)^{n} a c e /(c-e)\left(f_{3 n-1} a+f_{3 n} e\right)\right)\left((-1)^{n} a e /\left(f_{3 n-2} a+f_{3 n-1} e\right)\right)}{(-1)^{n} a e /\left(f_{3 n-1} a+f_{3 n} e\right)+(-1)^{n} a e /\left(f_{3 n-2} a+f_{3 n-1} e\right)} \\
& =\frac{\left((-1)^{n} a c e /(c-e)\left(f_{3 n-1} a+f_{3 n} e\right)\right)\left(1 /\left(f_{3 n-2} a+f_{3 n-1} e\right)\right)}{1 /\left(f_{3 n-1} a+f_{3 n} e\right)+1 /\left(f_{3 n-2} a+f_{3 n-1} e\right)}=\frac{(-1)^{n} a c e /(c-e)}{\left[f_{3 n-2} a+f_{3 n-1} e+f_{3 n-1} a+f_{3 n} e\right]} \\
& =\frac{(-1)^{n} a c e}{(c-e)\left(f_{3 n} a+f_{3 n+1} e\right)}, \\
y_{6 n+2} & =\frac{x_{6 n+1} x_{6 n-1}}{-y_{6 n}+x_{6 n-1}}=\frac{\left((-1)^{n} d f /\left(f_{3 n} b+f_{3 n+1} f\right)\right)\left((-1)^{n} b f /\left(f_{3 n-1} b+f_{3 n} f\right)\right)}{-(-1)^{n} d f /\left(f_{3 n-1} b+f_{3 n} f\right)+(-1)^{n} b f /\left(f_{3 n-1} b+f_{3 n} f\right)} \\
& =\frac{\left((-1)^{n} d f /\left(f_{3 n} b+f_{3 n+1} f\right)\right)\left(b /\left(f_{3 n-1} b+f_{3 n} f\right)\right)}{\left(-d /\left(f_{3 n-1} b+f_{3 n} f\right)+b /\left(f_{3 n-1} b+f_{3 n} f\right)\right.} \\
& =\frac{(-1)^{n} b d f}{\left(f_{3 n} b+f_{3 n+1} f\right)\left(f_{3 n-1} b+f_{3 n} f\right)\left[-d /\left(f_{3 n-1} b+f_{3 n} f\right)+b /\left(f_{3 n-1} b+f_{3 n} f\right)\right]} \frac{(-1)^{n} b d f}{\left(f_{3 n} b+f_{3 n+1} f\right)[-d+b]} \\
& =\frac{(-1)^{n} b d f}{(b-d)\left(f_{3 n} b+f_{3 n+1} f\right)} . \quad
\end{aligned}
$$

Thus, the other relations can be proven in a similar way. Hence the proof is complete.

2.4. The Fourth System: $x_{n+1}=y_{n} y_{n-2} /\left(x_{n-1}+y_{n-2}\right)$, $y_{n+1}=x_{n} x_{n-2} /\left(-y_{n-1}-x_{n-2}\right)$. In this subsection, we analyze the solutions of the system of the following two difference equations.

$$
\begin{aligned}
& x_{n+1}=\frac{y_{n} y_{n-2}}{x_{n-1}+y_{n-2}}, \\
& y_{n+1}=\frac{x_{n} x_{n-2}}{-y_{n-1}-x_{n-2}}, \\
& n=0,1, \ldots,
\end{aligned}
$$

with the initial conditions $x_{-2}, x_{-1}, x_{0}$ and $y_{-2}, y_{-1}, y_{0}$ being nonzero positive real numbers.

Theorem 5. Let $\left\{x_{n}, y_{n}\right\}_{n=-1}^{+\infty}$ be solutions of system (28). Then $\left\{x_{n}\right\}_{n=-1}^{+\infty}$ and $\left\{y_{n}\right\}_{n=-1}^{+\infty}$ are given by the following formulas for $n=0,1,2, \ldots$ :

$$
\begin{aligned}
x_{6 n-2} & =\frac{a c}{\left(f_{3 n-2} a+f_{3 n-1} e\right)}, \\
x_{6 n-1} & =\frac{b f}{\left(f_{3 n-1} b+f_{3 n} f\right)}, \\
x_{6 n} & =\frac{a e}{\left(f_{3 n-1} a+f_{3 n} e\right)}, \\
x_{6 n+1} & =\frac{d f}{\left(f_{3 n} b+f_{3 n+1} f\right)}, \\
x_{6 n+2} & =\frac{-a c e}{(c+e)\left(f_{3 n} a+f_{3 n+1} e\right)},
\end{aligned}
$$

$$
\begin{aligned}
x_{6 n+3} & =\frac{-b d f}{(b+d)\left(f_{3 n+1} b+f_{3 n+2} f\right)}, \\
y_{6 n-2} & =\frac{b f}{\left(f_{3 n-2} b+f_{3 n-1} f\right)}, \\
y_{6 n-1} & =\frac{a e}{\left(f_{3 n-2} a+f_{3 n-1} e\right)}, \\
y_{6 n} & =\frac{d f}{\left(f_{3 n-1} b+f_{3 n} f\right)}, \\
y_{6 n+1} & =\frac{-a c e}{(c+e)\left(f_{3 n-1} a+f_{3 n} e\right)}, \\
y_{6 n+2} & =\frac{-b d f}{(b+d)\left(f_{3 n} b+f_{3 n+1} f\right)}, \\
y_{6 n+3} & =\frac{a c}{\left(f_{3 n} a+f_{3 n+1} e\right)},
\end{aligned}
$$

where $x_{0}=a, x_{-1}=b, x_{-2}=c, y_{0}=d, y_{-1}=e, y_{-2}=f$ and where $f_{n}$ is the Fibonacci Sequence with $f_{-2}=1, f_{-1}=0$.

Proof. For $n=0$ the result holds. By using mathematical induction now suppose that $n>0$ and that our assumption holds for $n-1$. That is,

$$
\begin{aligned}
& x_{6 n-8}=\frac{a c}{\left(f_{3 n-5} a+f_{3 n-4} e\right)}, \\
& x_{6 n-7}=\frac{b f}{\left(f_{3 n-4} b+f_{3 n-3} f\right)}, \\
& x_{6 n-6}=\frac{a e}{\left(f_{3 n-4} a+f_{3 n-3} e\right)},
\end{aligned}
$$




$$
\begin{aligned}
& x_{6 n-5}=\frac{d f}{\left(f_{3 n-3} b+f_{3 n-2} f\right)}, \\
& x_{6 n-4}=\frac{-a c e}{(c+e)\left(f_{3 n-3} a+f_{3 n-2} e\right)}, \\
& x_{6 n-3}=\frac{-b d f}{(b+d)\left(f_{3 n-2} b+f_{3 n-1} f\right)}, \\
& y_{6 n-8}=\frac{b f}{\left(f_{3 n-5} b+f_{3 n-4} f\right)}, \\
& y_{6 n-7}=\frac{a e}{\left(f_{3 n-5} a+f_{3 n-4} e\right)},
\end{aligned}
$$

$$
\begin{aligned}
& y_{6 n-6}=\frac{d f}{\left(f_{3 n-4} b+f_{3 n-3} f\right)}, \\
& y_{6 n-5}=\frac{-a c e}{(c+e)\left(f_{3 n-4} a+f_{3 n-3} e\right)}, \\
& y_{6 n-4}=\frac{-b d f}{(b+d)\left(f_{3 n-3} b+f_{3 n-2} f\right)}, \\
& y_{6 n-3}=\frac{a c}{\left(f_{3 n-3} a+f_{3 n-2} e\right)} .
\end{aligned}
$$

Now, it follows from system (28) substitution of the above equations that

$$
\begin{aligned}
& x_{6 n-2}=\frac{y_{6 n-3} y_{6 n-5}}{x_{6 n-4}+y_{6 n-5}}=\frac{\left(a c /\left(f_{3 n-3} a+f_{3 n-2} e\right)\right)\left(-a c e /(c+e)\left(f_{3 n-4} a+f_{3 n-3} e\right)\right)}{-a c e /(c+e)\left(f_{3 n-3} a+f_{3 n-2} e\right)+-a c e /(c+e)\left(f_{3 n-4} a+f_{3 n-3} e\right)}=\frac{\left(a c /\left(f_{3 n-3} a+f_{3 n-2} e\right)\right)\left(1 /\left(f_{3 n-4} a+f_{3 n-3} e\right)\right)}{1 /\left(f_{3 n-3} a+f_{3 n-2} e\right)+1 /\left(f_{3 n-4} a+f_{3 n-3} e\right)} \\
& =\frac{a c}{\left(f_{3 n-3} a+f_{3 n-2} e\right)\left(f_{3 n-4} a+f_{3 n-3} e\right)\left[1 /\left(f_{3 n-3} a+f_{3 n-2} e\right)+1 /\left(f_{3 n-4} a+f_{3 n-3} e\right)\right]}=\frac{a c}{\left[\left(f_{3 n-4} a+f_{3 n-3} e\right)+\left(f_{3 n-3} a+f_{3 n-2} e\right)\right]} \\
& =\frac{a c}{\left(f_{3 n-2} a+f_{3 n-1} e\right)} \text {, } \\
& y_{6 n-2}=\frac{x_{6 n-3} x_{6 n-5}}{-y_{6 n-4}-x_{6 n-5}}=\frac{\left(-b d f /(b+d)\left(f_{3 n-2} b+f_{3 n-1} f\right)\right)\left(d f /\left(f_{3 n-3} b+f_{3 n-2} f\right)\right)}{-\left(-b d f /(b+d)\left(f_{3 n-3} b+f_{3 n-2} f\right)\right)-d f /\left(f_{3 n-3} b+f_{3 n-2} f\right)} \\
& =\frac{\left(-b d f /(b+d)\left(f_{3 n-2} b+f_{3 n-1} f\right)\right)\left(1 /\left(f_{3 n-3} b+f_{3 n-2} f\right)\right)}{-\left(-b /(b+d)\left(f_{3 n-3} b+f_{3 n-2} f\right)\right)-1 /\left(f_{3 n-3} b+f_{3 n-2} f\right)}=\frac{-b d f /(b+d)\left(f_{3 n-2} b+f_{3 n-1} f\right)}{b /(b+d)-1}=\frac{-b d f}{(b+d)\left(f_{3 n-2} b+f_{3 n-1} f\right)[b /(b+d)-1]} \\
& =\frac{-b d f}{\left(f_{3 n-2} b+f_{3 n-1} f\right)[b-b-d]}=\frac{b f}{\left(f_{3 n-2} b+f_{3 n-1} f\right)} \text {, } \\
& x_{6 n}=\frac{y_{6 n-1} y_{6 n-3}}{x_{6 n-2}+y_{6 n-3}}=\frac{\left(a e /\left(f_{3 n-2} a+f_{3 n-1} e\right)\right)\left(a c /\left(f_{3 n-3} a+f_{3 n-2} e\right)\right)}{a c /\left(f_{3 n-2} a+f_{3 n-1} e\right)+a c /\left(f_{3 n-3} a+f_{3 n-2} e\right)}=\frac{\left(a e /\left(f_{3 n-2} a+f_{3 n-1} e\right)\right)\left(1 /\left(f_{3 n-3} a+f_{3 n-2} e\right)\right)}{1 /\left(f_{3 n-2} a+f_{3 n-1} e\right)+1 /\left(f_{3 n-3} a+f_{3 n-2} e\right)} \\
& =\frac{a e}{\left(f_{3 n-2} a+f_{3 n-1} e\right)\left(f_{3 n-3} a+f_{3 n-2} e\right)\left[1 /\left(f_{3 n-2} a+f_{3 n-1} e\right)+1 /\left(f_{3 n-3} a+f_{3 n-2} e\right)\right]}=\frac{a e}{\left[f_{3 n-3} a+f_{3 n-2} e+f_{3 n-2} a+f_{3 n-1} e\right]} \\
& =\frac{a e}{\left(f_{3 n-1} a+f_{3 n} e\right)} \text {, } \\
& y_{6 n}=\frac{x_{6 n-1} x_{6 n-3}}{-y_{6 n-2}-x_{6 n-3}}=\frac{\left(b f /\left(f_{3 n-1} b+f_{3 n} f\right)\right)\left(-b d f /(b+d)\left(f_{3 n-2} b+f_{3 n-1} f\right)\right)}{-b f /\left(f_{3 n-2} b+f_{3 n-1} f\right)-\left(-b d f /(b+d)\left(f_{3 n-2} b+f_{3 n-1} f\right)\right)} \\
& =\frac{\left(b f /\left(f_{3 n-1} b+f_{3 n} f\right)\right)\left(-d /(b+d)\left(f_{3 n-2} b+f_{3 n-1} f\right)\right)}{-1 /\left(f_{3 n-2} b+f_{3 n-1} f\right)-\left(-d /(b+d)\left(f_{3 n-2} b+f_{3 n-1} f\right)\right)} \\
& =\frac{-b d f}{(b+d)\left(f_{3 n-2} b+f_{3 n-1} f\right)\left(f_{3 n-1} b+f_{3 n} f\right)\left[-1 /\left(f_{3 n-2} b+f_{3 n-1} f\right)-\left(-d /(b+d)\left(f_{3 n-2} b+f_{3 n-1} f\right)\right)\right]} \\
& =\frac{-b d f}{\left[-(b+d)\left(f_{3 n-2} b+f_{3 n-1} f\right)\left(f_{3 n-1} b+f_{3 n} f\right) /\left(f_{3 n-2} b+f_{3 n-1} f\right)+d(b+d)\left(f_{3 n-2} b+f_{3 n-1} f\right)\left(f_{3 n-1} b+f_{3 n} f\right) /(b+d)\left(f_{3 n-2} b+f_{3 n-1} f\right)\right]} \\
& =\frac{-b d f}{\left[-(b+d)\left(f_{3 n-1} b+f_{3 n} f\right)+d\left(f_{3 n-1} b+f_{3 n} f\right)\right]}=\frac{-b d f}{\left[-b\left(f_{3 n-1} b+f_{3 n} f\right)-d\left(f_{3 n-1} b+f_{3 n} f\right)+d\left(f_{3 n-1} b+f_{3 n} f\right)\right]}=\frac{-b d f}{-b\left(f_{3 n-1} b+f_{3 n} f\right)} \\
& =\frac{d f}{\left(f_{3 n-1} b+f_{3 n} f\right)} \text {. }
\end{aligned}
$$

\section{Also, we obtain}

$$
\begin{aligned}
x_{6 n+2} & =\frac{y_{6 n+1} y_{6 n-1}}{x_{6 n}+y_{6 n-1}}=\frac{\left(-a c e /(c+e)\left(f_{3 n-1} a+f_{3 n} e\right)\right)\left(a e /\left(f_{3 n-2} a+f_{3 n-1} e\right)\right)}{a e /\left(f_{3 n-1} a+f_{3 n} e\right)+a e /\left(f_{3 n-2} a+f_{3 n-1} e\right)} \\
& =\frac{\left(-a c e /(c+e)\left(f_{3 n-1} a+f_{3 n} e\right)\right)\left(1 /\left(f_{3 n-2} a+f_{3 n-1} e\right)\right)}{1 /\left(f_{3 n-1} a+f_{3 n} e\right)+1 /\left(f_{3 n-2} a+f_{3 n-1} e\right)}
\end{aligned}
$$




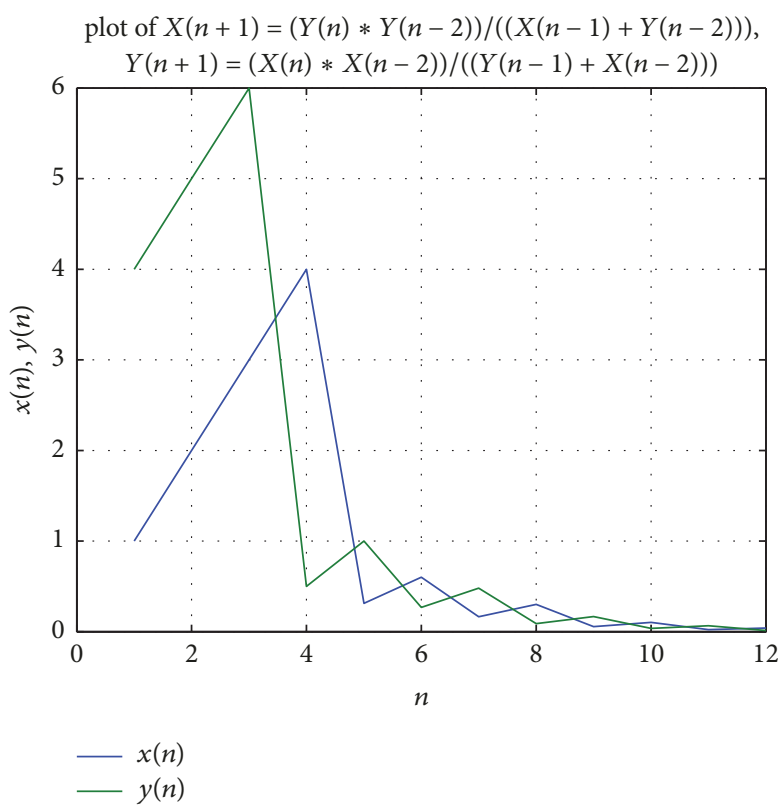

Figure 1: This figure shows the solution of (8).

$$
\begin{aligned}
& =\frac{-a c e /(c+e)}{\left(f_{3 n-1} a+f_{3 n} e\right)\left(f_{3 n-2} a+f_{3 n-1} e\right)\left[1 /\left(f_{3 n-1} a+f_{3 n} e\right)+1 /\left(f_{3 n-2} a+f_{3 n-1} e\right)\right]} \\
& =\frac{-a c e /(c+e)}{\left[f_{3 n-2} a+f_{3 n-1} e+f_{3 n-1} a+f_{3 n} e\right]}=\frac{-a c e}{(c+e)\left(f_{3 n} a+f_{3 n+1} e\right)} \\
y_{6 n+2} & =\frac{x_{6 n+1} x_{6 n-1}}{-y_{6 n}-x_{6 n-1}}=\frac{\left(d f /\left(f_{3 n} b+f_{3 n+1} f\right)\right)\left(b f /\left(f_{3 n-1} b+f_{3 n} f\right)\right)}{-d f /\left(f_{3 n-1} b+f_{3 n} f\right)-b f /\left(f_{3 n-1} b+f_{3 n} f\right)}=\frac{\left(d f /\left(f_{3 n} b+f_{3 n+1} f\right)\right)\left(b /\left(f_{3 n-1} b+f_{3 n} f\right)\right)}{-d /\left(f_{3 n-1} b+f_{3 n} f\right)-b /\left(f_{3 n-1} b+f_{3 n} f\right)} \\
& =\frac{b d f}{\left(f_{3 n} b+f_{3 n+1} f\right)\left(f_{3 n-1} b+f_{3 n} f\right)\left[-d /\left(f_{3 n-1} b+f_{3 n} f\right)-b /\left(f_{3 n-1} b+f_{3 n} f\right)\right]}=\frac{b d f}{\left(f_{3 n} b+f_{3 n+1} f\right)[-d-b]} \\
& =\frac{-b d f}{(b+d)\left(f_{3 n} b+f_{3 n+1} f\right)} .
\end{aligned}
$$

Thus, the other relations can be proven in a similar way. Hence the proof is complete.

\section{Numerical Examples}

To illustrate the results of foregoing sections and to support our theoretical discussions, we take into account several interesting numerical examples in this section.

Example 1 (first system: $x_{n+1}=y_{n} y_{n-2} /\left(x_{n-1}+y_{n-2}\right)$, $\left.y_{n+1}=x_{n} x_{n-2} /\left(y_{n-1}+x_{n-2}\right)\right)$. Figure 1 considers the solution of the difference equations system (8) with the initial conditions $x_{-2}=1, x_{-1}=2, x_{0}=3$ and $y_{-2}=4, y_{-1}=5$, $y_{0}=6$.
Example 2 (second system: $x_{n+1}=y_{n} y_{n-2} /\left(x_{n-1}+y_{n-2}\right)$, $\left.y_{n+1}=x_{n} x_{n-2} /\left(y_{n-1}-x_{n-2}\right)\right)$. Figure 2 indicates the solution of the difference equation system (17) with the initial conditions $x_{-2}=4, x_{-1}=7, x_{0}=2$ and $y_{-2}=2, y_{-1}=5, y_{0}=1$.

Example 3 (third system: $x_{n+1}=y_{n} y_{n-2} /\left(x_{n-1}+y_{n-2}\right)$, $\left.y_{n+1}=x_{n} x_{n-2} /\left(-y_{n-1}+x_{n-2}\right)\right)$. Figure 3 considers the solution of the difference equation system (23) with the initial conditions $x_{-2}=4, x_{-1}=7, x_{0}=2$ and $y_{-2}=2, y_{-1}=5$, $y_{0}=1$.

Example 4 (fourth system: $x_{n+1}=y_{n} y_{n-2} /\left(x_{n-1}+y_{n-2}\right)$, $\left.y_{n+1}=x_{n} x_{n-2} /\left(-y_{n-1}-x_{n-2}\right)\right)$. Figure 4 shows the solution of the difference equation system (28) with the initial conditions $x_{-2}=4, x_{-1}=7, x_{0}=2$ and $y_{-2}=2, y_{-1}=5, y_{0}=1$. 


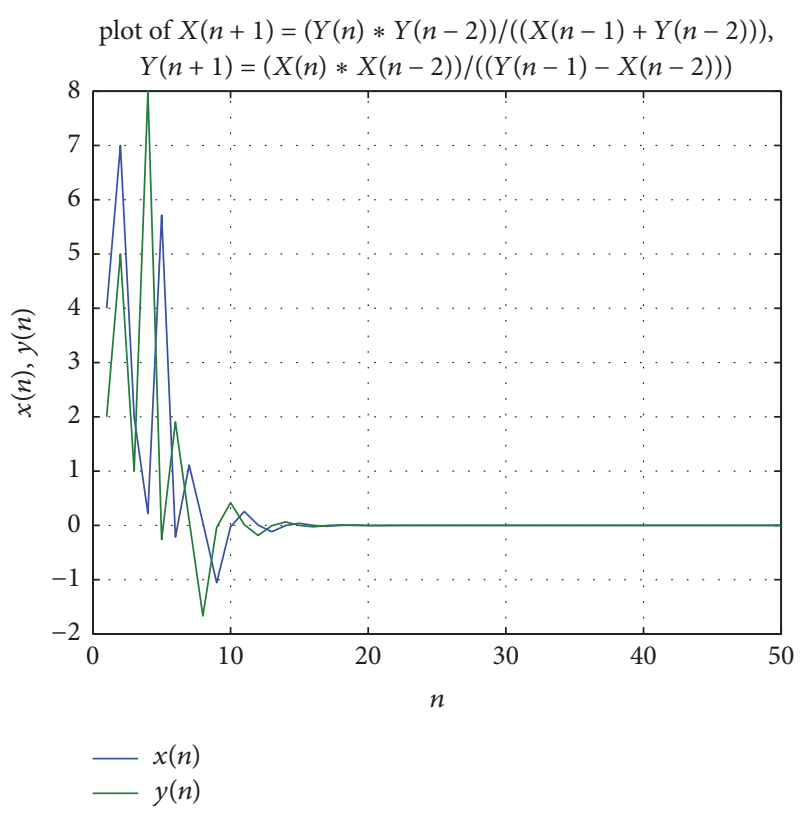

FIgURE 2: This figure shows the solution of (17).

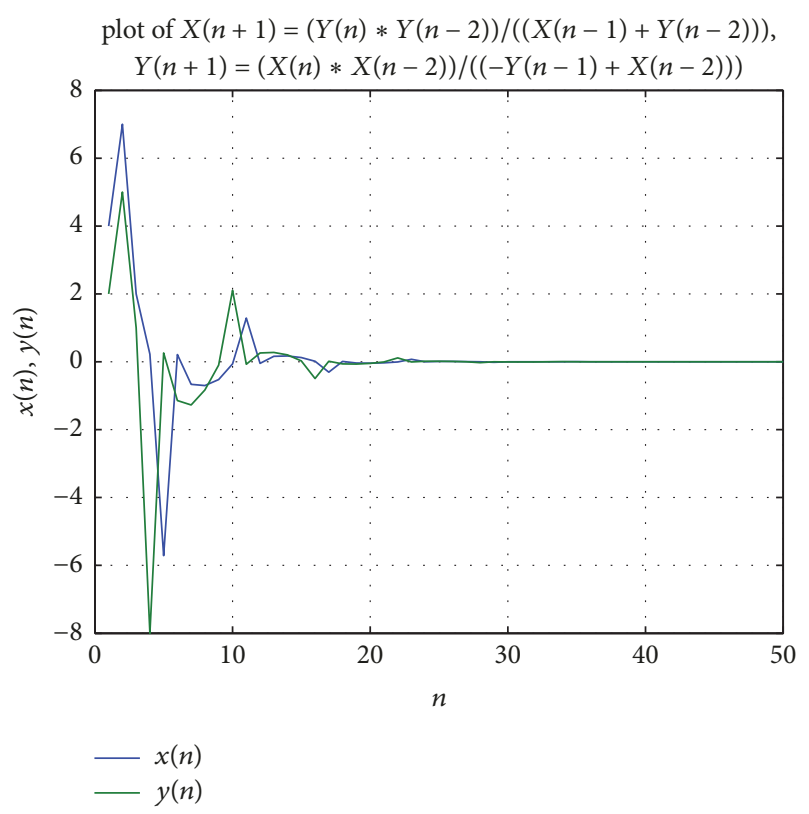

Figure 3: This figure shows the solution of (23).

\section{Conclusion}

In this analysis, we oversee the structure of the solutions of four cases of the difference equations system $x_{n+1}=$ $y_{n} y_{n-2} /\left(x_{n-1}+y_{n-2}\right), y_{n+1}=x_{n} x_{n-2} /\left( \pm y_{n-1} \pm x_{n-2}\right)$. Additionally, some behavior of the solutions such as boundedness is investigated. Subsequently, some numerical examples are displayed by presenting some numerical values for the initial values per case and figures provided to justify the behavior of the obtained solutions in the case of numerical examples.

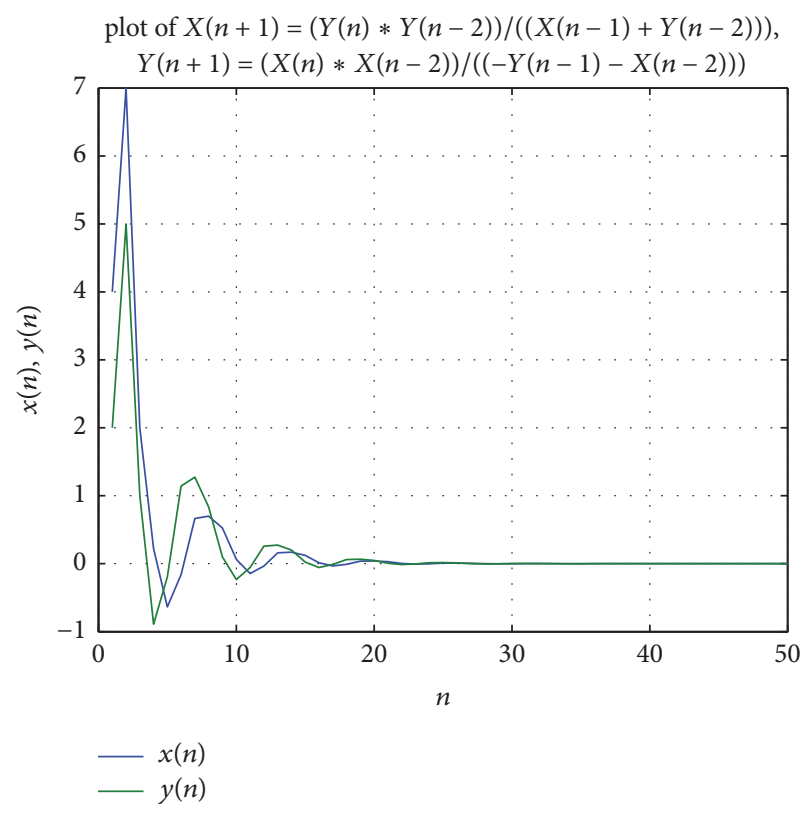

Figure 4: This figure shows the solution of (28).

\section{Conflicts of Interest}

The authors declare that there are no conflicts of interest regarding the publication of this paper.

\section{Acknowledgments}

This work is financially supported by UKM Grant DIP2017-011 and Ministry of Education Malaysia Grant FRGS/1/ 2017/STG06/UKM/01/1.

\section{References}

[1] A. Asiri, M. M. El-Dessoky, and E. M. Elsayed, "Solution of a third order fractional system of difference equations," Journal of Computational Analysis and Applications, vol. 24, no. 3, pp. 444-453, 2018.

[2] Q. Din, M. N. Qureshi, and A. Q. Khan, "Dynamics of a fourth-order system of rational difference equations," Advances in Difference Equations, vol. 2012, 15 pages, 2012.

[3] M. M. El-Dessoky, "The form of solutions and periodicity for some systems of third-order rational difference equations," Mathematical Methods in the Applied Sciences, vol. 39, no. 5, pp. 1076-1092, 2016.

[4] M. M. El-Dessoky, A. Khaliq, and A. Asiri, "On some rational systems of difference equations," Journal of Nonlinear Sciences and Applications, vol. 11, no. 01, pp. 49-72, 2017.

[5] M. M. El-Dessoky, E. M. Elsayed, and M. Alghamdi, "Solutions and periodicity for some systems of fourth order rational difference equations," Journal of Computational Analysis and Applications, vol. 18, no. 1, pp. 179-194, 2015.

[6] M. El-Moneam, "On the dynamics of the solutions of the rational recursive sequences," British Journal of Mathematics and Computer Science, vol. 5, no. 5, pp. 654-665, 2015.

[7] M. M. El-Dessoky and E. M. Elsayed, "On the solutions and periodic nature of some systems of rational difference 
equations," Journal of Computational Analysis and Applications, vol. 18, no. 2, pp. 206-218, 2015.

[8] E. M. Elsayed and A. Alghamdi, "The form of the solutions of nonlinear difference equations systems," Journal of Nonlinear Sciences and Applications. JNSA, vol. 9, no. 5, pp. 3179-3196, 2016.

[9] E. M. Elsayed, A. Alotaibi, and H. A. Almaylabi, "On a solutions of fourth order rational systems of difference equations," Journal of Computational Analysis and Applications, vol. 22, no. 7, pp. 1298-1308, 2017.

[10] A. S. Kurbanli, "On the behavior of solutions of the system of rational difference equations," World Applied Sciences Journal, vol. 10, pp. 1344-1350, 2010.

[11] M. Mansour, M. M. El-Dessoky, and E. M. Elsayed, "On the solution of rational systems of difference equations," Journal of Computational Analysis and Applications, vol. 15, no. 5, pp. 967976, 2013.

[12] N. Touafek and E. M. Elsayed, "On a second order rational systems of difference equations," Hokkaido Mathematical Journal, vol. 44, no. 1, pp. 29-45, 2015.

[13] A. Gelisken and M. Kara, "Some general systems of rational difference equations," Journal of Difference Equations, vol. 2015, Article ID 396757, 7 pages, 2015.

[14] T. F. Ibrahim and N. Touafek, "On a third order rational difference equation with variable coefficients," Dynamics of Continuous, Discrete and Impulsive Systems. Series B. Applications and Algorithms, vol. 20, no. 2, pp. 251-264, 2013.

[15] A. Q. Khan, M. N. Qureshi, and Q. Din, "Asymptotic behavior of an anti-competitive system of rational difference equations," Life Science Journal, vol. 11, no. 7, article no. 3, pp. 16-20, 2014.

[16] V. Khuong and T. Thai, "Asymptotic behavior of the solutions of system of difference equations of exponential form," International Journal of Difference Equations, vol. 2014, Article ID 936302, 6 pages, 2014

[17] H. Ma and H. Feng, "On positive solutions for the rational difference equation systems $x_{n+1}=A / x_{n} y_{n}^{2}$ and $y_{n+1}=$ $B y_{n} / x_{n-1} y_{n-1}$, International Scholarly Research Notices, vol. 2014, Article ID 857480, 4 pages, 2014.

[18] O. Ocalan, "Global dynamics of a non-autonomous rational difference equation," Journal of Applied Mathematics and Informatics, vol. 32, no. 5-6, pp. 843-848, 2014.

[19] D. T. Tollu, Y. Yazlik, and N. Taskara, "On fourteen solvable systems of difference equations," Applied Mathematics and Computation, vol. 233, pp. 310-319, 2014.

[20] I. Yalcinkaya, "On the global asymptotic behavior of a system of two nonlinear difference equations," Ars Combinatoria, vol. 95, pp. 151-159, 2010. 


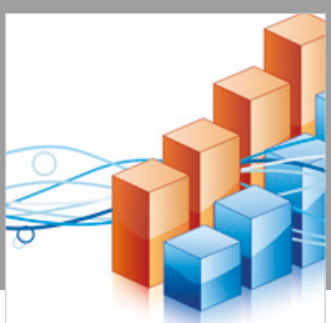

Advances in

Operations Research

\section{-n-m}
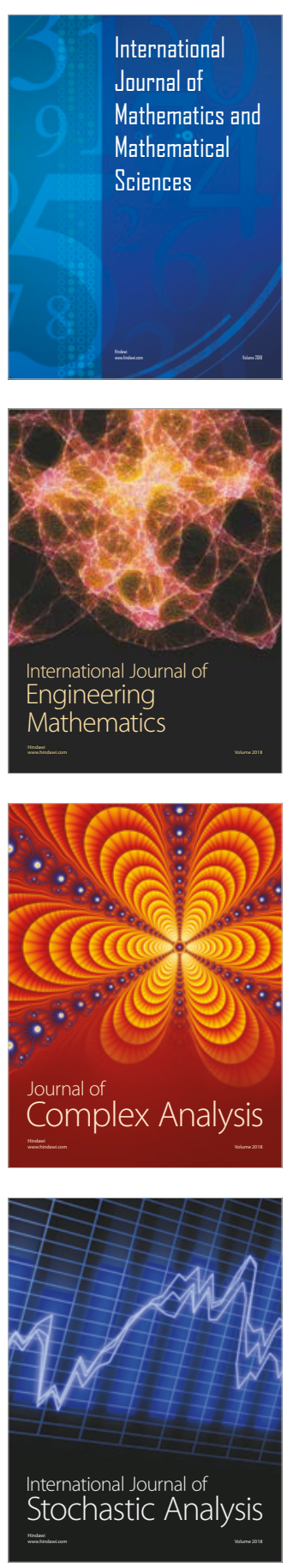
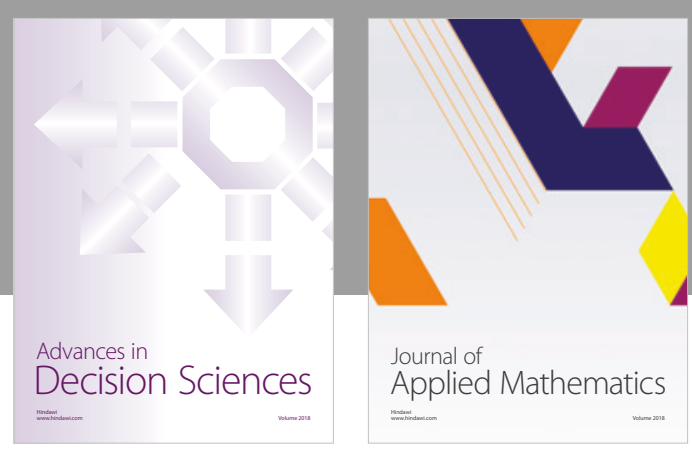

Journal of

Applied Mathematics
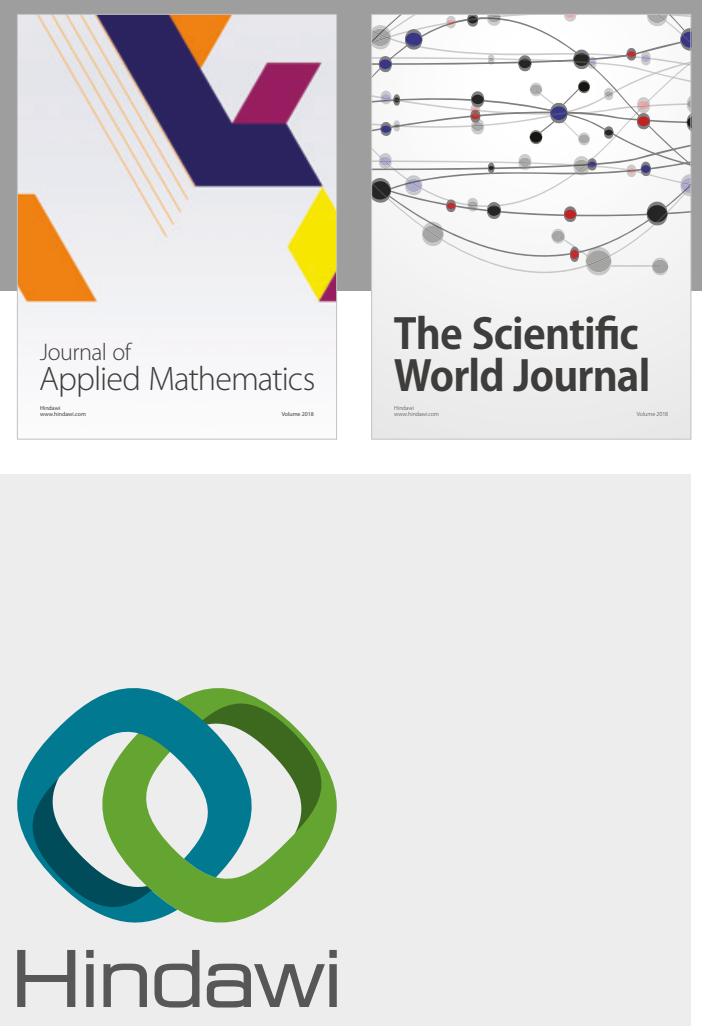

Submit your manuscripts at

www.hindawi.com

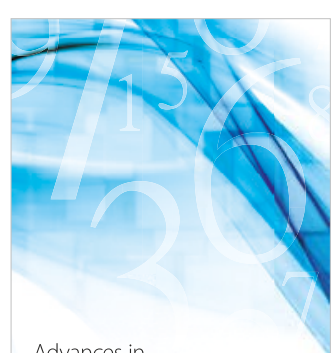

Advances in
Numerical Analysis
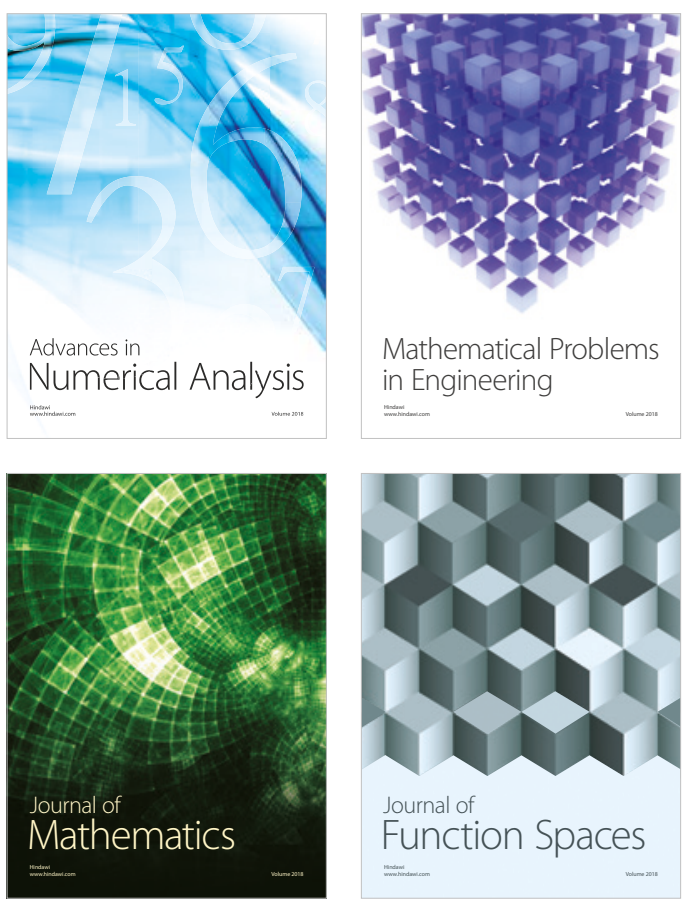

Mathematical Problems in Engineering

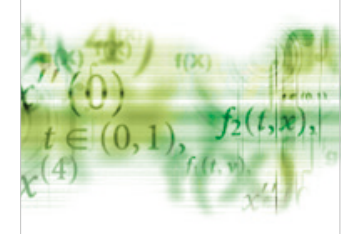

International Journal of

Differential Equations

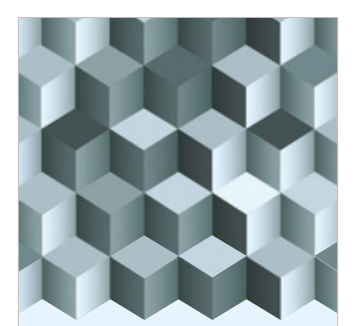

Journal of

Function Spaces

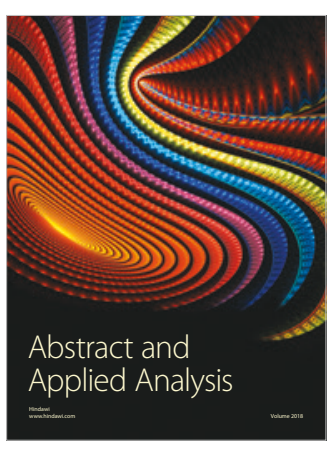

The Scientific

World Journal

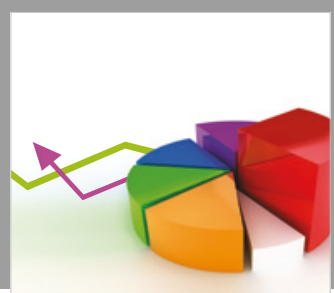

Journal of

Probability and Statistics
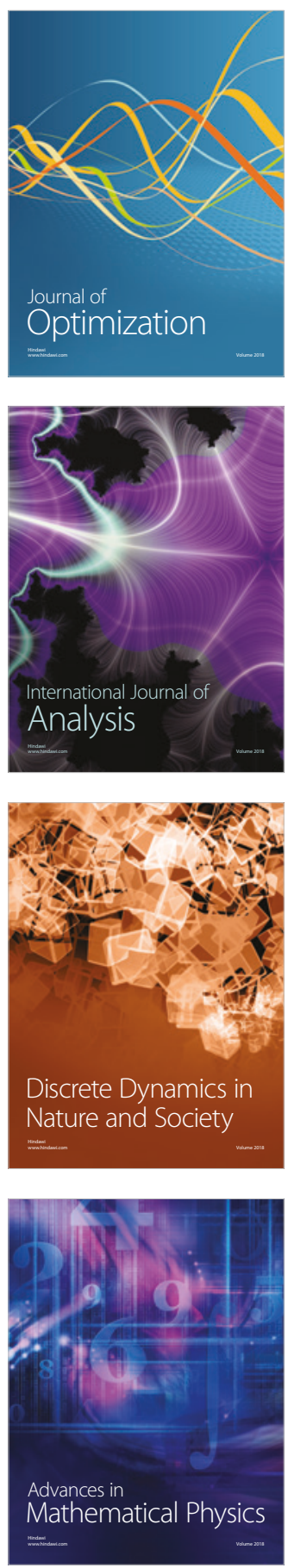